\title{
Simulação de leito empacotado com misturas binárias de esferas de diâmetros diferentes empregando correlações e o Método de Elementos Discretos
}

Simulation of packed bed with binary mixtures of particles differing in size using correlations and the Discrete Elements Method

Simulación de lecho empacado con mezclas binarias de esferas de diferentes diámetros usando correlaciones y el Método de Elementos Discretos

Recebido: 03/08/2021 | Revisado: 09/08/2021 | Aceito: 16/08/2021 | Publicado: 18/08/2021

Daiane Bortolote Ferreira

ORCID: https://https://orcid.org/0000-0003-1718-3942 Universidade Estadual Paulista Júlio de Mesquita Filho, Brasil

E-mail: daia.bferreira@hotmail.com

Ricardo Correa de Santana ORCID: https://https://orcid.org/0000-0002-6022-5821 Universidade Federal de Uberlândia, Brasil E-mail: ricardo@feq.ufu.br

Marcos Antônio de Souza Barrozo ORCID: https://https://orcid.org/0000-0002-8873-162X Universidade Federal de Uberlândia, Brasil E-mail: masbarrozo@ufu.br

Davi Leonardo de Souza

ORCID: https://https://orcid.org/0000-0002-1995-9057 Universidade Federal do Triângulo Mineiro, Brasil E-mail: davi.souza@uftm.edu.br José Luiz Vieira Neto

ORCID: https://https://orcid.org/0000-0003-0736-3974 Universidade Federal do Triângulo Mineiro, Brasil E-mail: jose.neto@uftm.edu.br

Kassia Graciele dos Santos

ORCID: https://https://orcid.org/0000-0001-7452-6900

Universidade Federal do Triângulo Mineiro, Brasil

E-mail: kassia.santos@uftm.edu.br

\begin{abstract}
Resumo
O leito de jorro é um equipamento altamente influenciado pela porosidade do leito estático. Assim, este trabalho teve o objetivo de empregar correlações da literatura e o método de elementos discretos na simulação do empacotamento de leitos de monopartículas e misturas binárias de esferas de 1 e $4 \mathrm{~mm}$. A porosidade mínima experimental foi 0,267, sendo que a correlação de Dias foi a que mais se aproximou do valor experimental e que conseguiu distinguir adequadamente os regimes de empacotamento de ocupação e preenchimento. Quanto às simulações empregando o Método de Elementos Discretos, a calibração dos parâmetros do modelo de força de contato de Hertz-Mindlin mostrou que o coeficiente de atrito estático partícula-partícula é a variável que mais influencia a operação de empacotamento, seguido pelo coeficiente de atrito de rolamento partícula-partícula, enquanto o coeficiente de atrito de rolamento partícula-parede não influenciou a porosidade do leito. As simulações representaram adequadamente a transição entre os regimes de ocupação e enchimento que regem o empacotamento.
\end{abstract}

Palavras-chave: Porosidade mínima; Mistura de partículas; Empacotamento; DEM; Calibração paramétrica.

\begin{abstract}
The spouted bed is an equipment highly influenced by the porosity of the static bed. Thus, this work aimed to employ literature correlations and the discrete element method to simulate the packing of monoparticle beds and binary mixtures of 1 and $4 \mathrm{~mm}$ spheres. The experimental minimum porosity was 0.267 , and the Dias correlation agreed well with experimental data and was able to distinguish the occupation and filling packing regimes adequately. Regarding the simulations using the Discrete Element Method, the parameters calibration of the Hertz-Mindlin contact force model showed that the particle-particle static friction coefficient is the factor that most influences the packing, followed by the particle-to-particle friction coefficient bearing friction coefficient, while the particle-to-wall bearing friction coefficient did not influence the bed voidage. The simulations adequately represented the transition between the occupation and filling regimes that govern packing.
\end{abstract}

Keywords: Minimum porosity; Particle mixture; Packing; DEM; Parametric calibration. 


\begin{abstract}
Resumen
El lecho jorrado es un equipo muy influenciado por la porosidad del lecho estático. Por lo tanto, este trabajo tuvo como objetivo emplear correlaciones de la literatura y el método de elementos discretos para simular el empaquetamiento de lechos de monopartículas y mezclas binarias de esferas de 1 y $4 \mathrm{~mm}$. La porosidad mínima experimental fue 0.267, y la correlación Dias fue la más cercana al valor experimental y fue capaz de distinguir adecuadamente los regímenes de empaquetamiento de ocupación y llenado. En cuanto a las simulaciones que utilizan el Método de Elementos Discretos, la calibración de los parámetros del modelo de fuerza de contacto de HertzMindlin mostró que el coeficiente de fricción estática partícula-partícula es la variable que más influye en la operación de empaque, seguido del coeficiente de fricción del rodamiento partícula-partícula, mientras que el coeficiente de fricción del rodamiento de partícula-pared no influyó en la porosidad del lecho. Las simulaciones representaron adecuadamente la transición entre los regímenes de ocupación y llenado que rigen el empaque.
\end{abstract}

Palabras clave: Porosidad mínima; Mezcla de partículas, Empaquetamiento, DEM, Calibración paramétrica.

\title{
1. Introdução
}

Algumas aplicações do leito de jorro envolvem um conjunto de partículas de tamanho heterogêneo ou mesmo mistura de diferentes partículas. No caso da pirólise rápida de biomassa, material inerte, geralmente areia, é adicionado à biomassa para aumentar a estabilidade do leito de jorro, além de facilitar o aquecimento da biomassa no leito (Santos et al., 2013). No entanto, o conhecimento do comportamento fluidodinâmico de uma mistura de partículas no leito de jorro é fundamental para o projeto dos reatores de pirólise, que devem operar numa condição em que ocorra a mistura das partículas.

Simulações computacionais de leito de jorro operando com leito de monopartículas são usuais e há uma metodologia bem estabelecida que leva a resultados simulados por CFD muito próximos aos experimentais (Vieira Neto et al., 2008; Santos et al., 2009). Entretanto, simulações computacionais de leito de jorro com mistura binária ou multicomponente não são comuns, sendo encontrados poucos trabalhos na literatura (Santos et al., 2015). É necessário estudar a aplicabilidade de alguns modelos próprios para misturas, principalmente os que regulamentam a distância entre as partículas e o limite máximo de empacotamento.

No entanto, algumas das condições iniciais requeridas para simular a fluidodinâmica de misturas de partículas em leito de jorro não são encontradas nos trabalhos experimentais relevantes sobre o assunto, como os valores de porosidade da mistura.

O leito de jorro é um equipamento altamente influenciado pela porosidade do leito estático. Leitos com maior empacotamento oferecem maior resistência ao escoamento das partículas, chegando a maiores picos de queda de pressão na curva característica do leito de jorro. No entanto, se a porosidade na região anular é menor, o escoamento do gás concentra-se mais na região de jorro e assim, a vazão necessária para iniciar o jorro é menor. Assim, conhecer a porosidade de misturas binárias é uma condição inicial essencial para a modelagem e simulação do leito de jorro. Dessa forma, observou-se a necessidade de estudar a relação entre a composição de uma mistura e sua porosidade.

A porosidade de um leito não está somente relacionada à composição das misturas binárias, mas também às forças de fricção a que as partículas são submetidas e ao método de empacotamento.

O empacotamento de misturas binárias de partículas foi analisado geometricamente por Graton e Fraser (1935) e analiticamente por Yu e Standish (1988). Foi observada a existência de dois mecanismos que influenciam diretamente na porosidade da mistura.

O mecanismo de enchimento ocorre quando a estrutura ou esqueleto formado pelas partículas maiores não se altera devido à adição de outras partículas menores, conforme mostra a Figura 1a. Para maiores razões de tamanho entre as partículas, ocorre o mecanismo de ocupação, no qual ambos os componentes influenciam a estrutura de empacotamento e consequentemente a porosidade da mistura no leito, como mostra a Figura 1b (Yu \& Standish, 1991). 
Figura 1. Mecanismo de enchimento (a) e de ocupação (b), em que as linhas pontilhadas representam as ligações entre o componente que controla a mistura (Yu \& Standish, 1991).

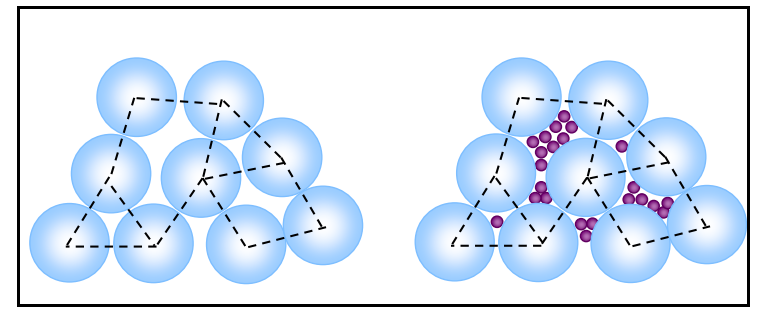

(a)

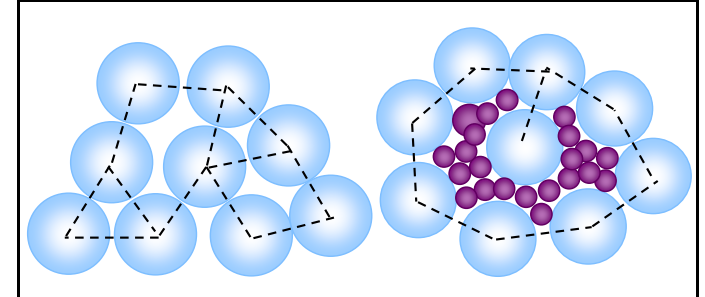

(b)

Fonte: Santos (2011) adaptado de Yu e Standish (1991).

Muitos trabalhos da literatura buscam investigar os mecanismos que regem a variação da porosidade de leitos empacotados, visto que é de conhecimento geral que a fração de vazios varia de acordo com a distribuição de tamanho de partículas que compõe o leito.

Uma grande variação do volume específico constitui uma grande interação interpartículas e, consequentemente, uma redução da porosidade, devido à mistura de partículas de tamanhos diferentes.

$\mathrm{Na}$ literatura, encontram-se alguns trabalhos sobre a porosidade de leitos contendo misturas de partículas. Dias, et al. (2004) estudaram a variação da porosidade com a composição de misturas binárias de esferas de vidro, para diferentes razões entre os diâmetros das partículas ( $\delta$ ) que compõe a mistura (entre 0,1 e 0,0375). Um modelo linear de mistura foi adotado para predizer a porosidade de cada fração de partículas na mistura binária. Eles ressaltaram que os desvios entre os dados experimentais e do modelo podem ter sido causados pelo efeito de parede, que é mais pronunciado em misturas com alta fração volumétrica de partículas maiores. Além disso, misturas com razão entre os diâmetros de partículas maiores que 0,0035 induzem a um efeito similar ao de parede, na interface entre as partículas maiores e menores. As predições de modelos de porosidade podem ficar comprometidas se não contabilizarem a possibilidade dos poros entre as partículas maiores serem preenchidos pelas partículas menores, dando origem a um leito com baixa porosidade. Desta forma, Dias et al. (2004) empregaram uma função de correção válida para a região em que a porosidade é mínima.

Mota et al. (2004) também incorporaram funções de correção ao modelo de empacotamento linear, como proposto por Yu et al. (1996).

As simulações computacionais de sistemas envolvendo sólidos particulados têm contribuído de forma significativa na otimização de processos e podem ser aplicadas a diversas operações, tais como fluidização (Araújo \& Santos, 2017), fluidodinâmica de leitos de jorro (Santos et al., 2015; Santos et al., 2017; Batista Jr et al., 2019), secadores e granuladores de tambor rotativo (Silvério et al., 2014; Gravena et al., 2019), secador solar de leito fixo (Stoppe et al, 2020; Rocha et al., 2020), aquecimento de forno solar (Faria et al., 2020), granulador de prato (Vieira Neto et al., 2017), câmara de separação gravitacional (Almeida et al., 2020; Mangucci et al., 2020), ciclones (Lacerda et al., 2005), hidrociclones (Ullmann et al., 2021), silos de descarga (Paula et al, 2020).

O Método de Elementos Discretos (DEM) tem sido utilizado com sucesso na simulação do empacotamento de partículas. Segundo Hlosta et al. (2020), o teste de empacotamento pode ser utilizado para estimação inicial e otimização, a fim de encontrar uma combinação adequada de parâmetros para o modelo de forças de contato, que representam adequadamente a densidade bulk de um material.

Assim, o presente trabalho teve o objetivo de investigar a variação da porosidade de um leito empacotado com misturas de esferas de vidro de tamanhos diferentes, com diferentes composições. Além disso, a porosidade das misturas foi predita por correlações da literatura e também simulações computacionais, empregando o Modelo de Elementos Discretos 
(DEM). Nas simulações, estudou-se o efeito do coeficiente de atrito estático e de rolamento partícula-partícula e partículaparede sobre a porosidade do leito após o empacotamento livre de esferas de vidro de 0,004 $\mathrm{m}$. Após a calibração dos parâmetros as forças de contato, os coeficientes foram empregados na simulação de uma mistura de EV com 1mm e 4mm, na condição de empacotamento máximo, no qual a porosidade do leito com mistura binária de partículas é mínima.

Essas informações permitem prever as condições de porosidade de um leito estático e poderão ser utilizadas como condições iniciais para a simulação CFD de leitos de jorro operando com mistura de partículas.

\section{Método de Elementos Discretos (DEM)}

O método de elementos discretos (DEM) envolve a descrição do movimento de cada partícula no escoamento e a modelagem da colisão entre as partículas e os contornos rígidos, sendo o método mais adequado para lidar com problemas de natureza descontínua, e ao mesmo tempo com grandes níveis de deformação, como é o caso do escoamento granular.

A metodologia DEM e suas variantes estão bem estabelecidas e são descritas em detalhes por Cleary (2008), que utiliza o método da esfera macia (Cundall \& Strack, 1979). O modelo de contato usado para contabilizar as forças de interação entre duas esferas rígidas que pertencem a duas diferentes partículas, é baseado no modelo de Hertz-Mindlin, que caracteriza as interações por meio de três coeficientes: coeficiente de restituição, coeficiente de fricção estático e coeficiente de fricção de rolamento.

No modelo de colisão de Hertz-Mindlin, a componente normal da força é baseada na teoria de contato de Hertz (1882), enquanto a força tangencial é baseada no trabalho de Mindlin-Deresiewicz (Mindlin, 1949; Mindlin \& Deresiewicz, 1953).

Tanto no cálculo da força normal, quanto da tangencial, há um termo de amortecimento do sistema, relacionado ao coeficiente de restituição (Tsuji et al. 1992), que relaciona a velocidade de separação e a velocidade de aproximação de uma ou mais partículas em uma colisão.

A força normal $\left(F_{n}\right)$ é uma função da sobreposição na direção normal $\left(\delta_{n} k\right)$ dada por:

$$
F_{n}=\frac{4}{3} E^{*} \sqrt{R^{*}} \delta_{n}^{3 / 2}
$$

no qual o módulo Equivalente de Young $\left(E^{*}\right)$ e o raio equivalente $\left(R^{*}\right)$ são definidos como:

$$
\begin{aligned}
& \frac{1}{E^{*}}=\frac{\left(1-v_{i}^{2}\right)}{E_{i}}+\frac{\left(1-v_{j}^{2}\right)}{E_{j}} \\
& \frac{1}{R^{*}}=\frac{1}{R_{i}}+\frac{1}{R_{j}}
\end{aligned}
$$

onde $E_{i}, v_{i}, R_{j}$ e $E_{j}, v_{j}, R_{j}$ são o modulo de Young, razão de Poisson e o raio de cada partícula em contato.

Adicionalmente, há a força normal de amortecimento $\left(F_{n}^{d}\right)$ dada por:

$$
F_{n}^{d}=-2 \sqrt{\frac{5}{6}}\left[\frac{\ln e}{\sqrt{\ln ^{2} e+\pi^{2}}}\right] \sqrt{\left(2 E^{*} \sqrt{R^{*} \delta_{n}}\right) m^{*}} v_{n}^{\overline{r e l}}
$$

onde $m^{*}=\left(m_{1}^{-1}+m_{i}^{-1}\right)^{-1}$ é a massa equivalente, $v_{n}^{\text {rel }}$ é velocidade relativa na direção normal e $e$ é o coeficiente de restituição.

A força tangencial $F_{t}$ depende da sobreposição na direção tangencial $\left(\delta_{t}\right)$ e constante de rigidez $\left(S_{t}\right)$ :

$$
F_{t}=-\delta_{t} S_{t}=-\delta_{t} 8 G^{*} \sqrt{R^{*} \delta_{n}}
$$

no qual $G^{*}$ é o módulo cisalhante. 
A força de amortecimento tangencial, conhecida também como força de repulsão, é dada por:

$$
F_{t}^{d}=-2 \sqrt{\frac{5}{6}}\left[\frac{\ln e}{\sqrt{\ln ^{2} e+\pi^{2}}}\right] \sqrt{S_{t} m^{*}} v_{t}^{\text {rel }}
$$

sendo $v_{t}^{\overline{r e l}}$, a velocidade relativa tangencial.

A força tangencial é limitada pelo atrito estático definido por Coulomb $\left(\mu_{s} F_{n}\right)$, onde $\mu_{s}$ é o coeficiente de atrito estático.

O coeficiente de atrito de rolamento modela o efeito da rugosidade da superfície de partículas não esféricas. Para tal, o coeficiente de atrito de rolamento introduz um torque artificial que no modelo de contato que se opõe ao movimento de rolamento, como segue:

$$
\tau_{i}=-\mu_{r} F_{n} R_{i} \omega_{i} v
$$

no qual $\mu_{r}$ é o coeficiente de atrito de rolamento, $R_{i}$ é a distância entre o ponto de contato e o centro de massa da partícula e $\omega_{i}$ é a vetor unitário da velocidade angular no ponto de contato.

O modelo da força de contato requer a informação de ambos os coeficientes de atrito das partículas e de restituição. Estes parâmetros são de difícil obtenção experimental e dependem do tipo de partícula. O algoritmo do DEM é bastante simples e pode ser descrito conforme mostra ao esquema da Figura 2.

Figura 2. Etapas básicas do ciclo de cálculo do método de elementos discretos (DEM).

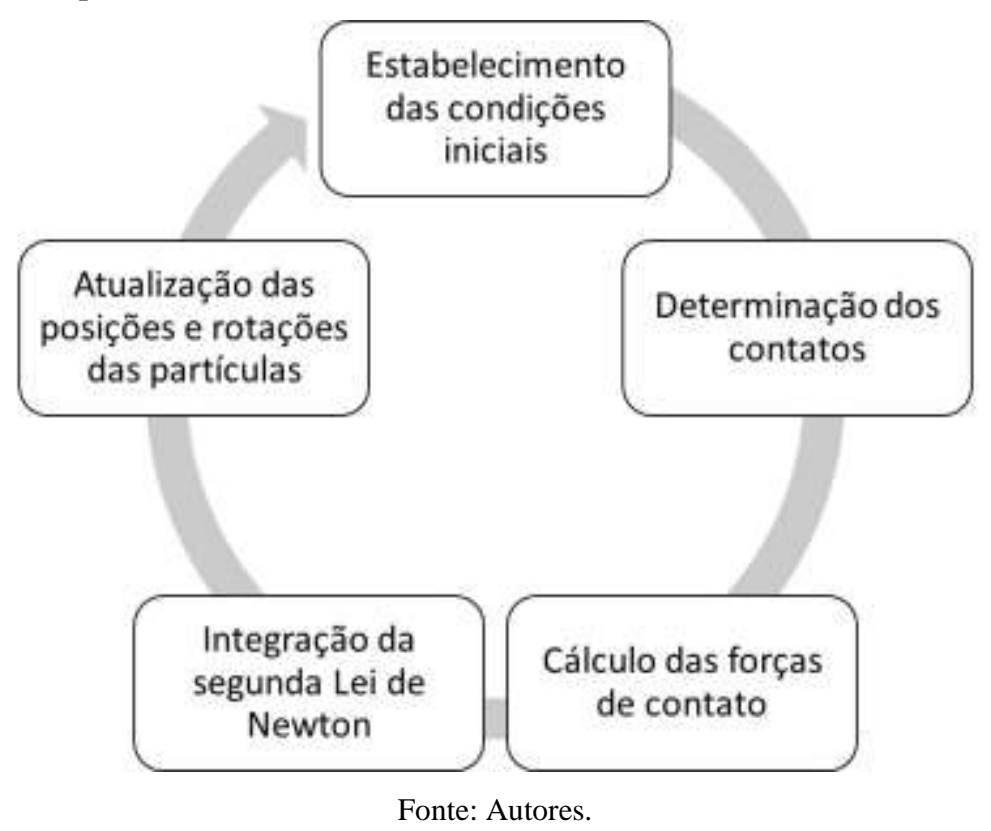

Segundo a Figura 2, a partir das condições iniciais e de contorno do problema, como velocidade de ar e partículas, bem como ponto e modo de injeção, define-se os modelos de contato partícula-partícula e partícula-parede, a fim de realizar o cálculo das forças de contato.

Desta forma, a equação do movimento da partícula, regida pela $2^{\mathrm{a}}$ Lei de Newton pode ser integrada em cada posição dentro do equipamento, num dado intervalo de tempo. São então atualizadas as posições e rotações das partículas, e incrementado o tempo, deforma a repetir o ciclo até que o tempo final de simulação seja atingido. 


\section{Metodologia}

\subsection{Procedimento experimental}

Este trabalho é de natureza quantitativa cujo suporte metodológico para os experimentos e simulações numéricas serão descritos a seguir (Pereira et al., 2018).

Para isso, foram utilizados dois tamanhos de esferas de vidro, sendo uma de diâmetro $\mathrm{D}=0,004 \mathrm{~m} \mathrm{e} \mathrm{d} \mathrm{=0,001} \mathrm{m,}$ ambas com densidade de $2480 \mathrm{~kg} / \mathrm{m}^{3}$. Foram preparadas misturas com as seguintes frações volumétricas das esferas maiores, $X_{D}: 0 ; 0,25 ; 0,50 ; 0,75 ; 0,85$ e 1 . Cada mistura foi preparada considerando a proporção mássica/volumétrica das diferentes partículas, estabelecida por $X_{D}$. As partículas foram pesadas e acrescentadas aleatoriamente a um recipiente cônico, similar ao cone do leito de jorro empregado neste estudo, até que fosse atingida a altura de leito estático de 0,08 m. As medidas de porosidade foram obtidas em triplicata, calculadas pela Eq. 8:

$$
\varepsilon=1-\frac{V}{V_{R}}
$$

em que $\varepsilon$ é a porosidade, $V$ é o volume real de partículas e $V_{R}$ é o volume do recipiente em que as partículas foram empacotadas, que neste caso era o mesmo tronco de cone utilizado nos experimentos de leito de jorro.

\subsection{Simulação empregando correlações da literatura}

A Tabela 1 apresenta as correlações empregadas neste trabalho para cálculo da porosidade da mistura de partículas em estudo.

Se um sistema composto de uma mistura de partículas for considerado de forma análoga a um sistema de soluções da termodinâmica em geral, então o volume da mistura (V) é definido em termos do volume específico parcial ( $\left.\mathrm{V}_{\mathrm{i}}\right)$, como mostra o Modelo A, presente na Tabela 1 (Yu \& Standish, 1988). No entanto, tratando-se de partículas sólidas, os mecanismos de empacotamento já mencionados influenciam de forma significativa o volume total da mistura. Sendo assim, surgiram outros modelos de empacotamento de misturas adequados a partículas esféricas, capazes de prever o efeito da razão de diâmetro entre as partículas e a também relacionar a fração de cada partícula na mistura com a porosidade total.

Neste trabalho foram empregados os modelos A, B, C e D dispostos na Tabela 1, que consideram como condição de contorno:

$$
\left\{\begin{array}{lll}
X_{D}=1 \rightarrow \varepsilon_{D}^{\left(X_{D}\right)}=\varepsilon_{D}^{(0)} & \text { e } & \varepsilon_{d}^{\left(X_{D}\right)}=1 \\
X_{D}=0 \rightarrow \varepsilon_{D}^{\left(X_{D}\right)}=1 & \text { e } & \varepsilon_{d}^{\left(X_{D}\right)}=\varepsilon_{d}^{(0)}
\end{array}\right.
$$

sendo $X_{D}$, a fração volumétrica da maior partícula na mistura; $\varepsilon_{D}^{(0)}$, a porosidade do leito composto apenas por partículas maiores e $\varepsilon_{d}^{(0)}$, a porosidade de um leito contendo apenas partículas menores. 
Tabela 1. Modelos para cálculo da porosidade de mistura de partículas esféricas.

Modelos de Porosidade
A- Yu e Standish (1988):

$$
V=\sum_{i=1}^{n} X_{i} \bar{V}_{i} ; \varepsilon=1-\frac{1}{V}
$$
$\bar{V}_{i}(i=1,2, \ldots, n):$ volume específico parcial do componente $\mathrm{i}$

\begin{tabular}{|c|c|}
\hline B- Yu et al. (1996) & $\begin{array}{c}\varepsilon=\varepsilon_{d}^{\left(X_{D}\right)} \varepsilon_{D}^{\left(X_{D}\right)} \\
\varepsilon_{D}^{\left(X_{D}\right)}=1-\left(1-\varepsilon_{D}^{(0)}\right) X_{\mathrm{D}} \cdot F\left(\delta, X_{D}\right) \\
\varepsilon_{d}^{\left(X_{D}\right)}=\varepsilon_{d}^{(0)}+\left(1-\varepsilon_{d}^{(0)}\right) X_{\mathrm{D}} \cdot f\left(\delta, X_{D}\right)\end{array}$ \\
\hline & $\begin{array}{c}F\left(\delta, X_{D}\right)=X_{D}^{\left[0,35-X_{D} F(\delta)\right]} ; \quad f\left(\delta, X_{D}\right)=X_{D}^{[f(\delta)-1]} \\
\varepsilon=\left\{1-\left(1-\varepsilon_{D}^{(0)}\right) X_{D}^{\left[1,35-X_{D} F(\delta)\right]}\right\} \cdot\left[\varepsilon_{d}^{(0)}+\left(1-\varepsilon_{d}^{(0)}\right) X_{D}^{[f(\delta)]}\right] \\
F(\delta)=\left\{0,27-\frac{1,55}{1+1 / \exp \left(\frac{\delta+0,06}{0,27}\right)}\right\} ; \quad f(\delta)=5-4 \sqrt{\delta}\end{array}$ \\
\hline C-Dias et al. (2004) & $\begin{array}{c}X_{D, \text { min }}=\frac{1-\varepsilon_{D}^{(0)}}{1-\varepsilon_{d}^{(0)} \varepsilon_{D}^{(0)}} ; \varepsilon=\frac{\varepsilon_{d}^{(0)}\left(1-X_{D}\right) \exp \left(1,2264 X_{D}^{1 / \sqrt{\delta}}\right)}{1-\varepsilon_{d}^{(0)} X_{D}}, X_{D} \leq X_{D, \text { min }} \\
\varepsilon=1-\frac{1-\varepsilon_{D}^{(0)}}{X_{D}}, \quad X_{D}>X_{D, \text { min }}\end{array}$ \\
\hline D- Yu et al. (1993) & $\begin{array}{l}V=\frac{V_{D} V_{d}}{V_{D}+V_{d}-1} ; \quad X_{D}=\frac{1-\varepsilon_{D}}{1-\varepsilon_{D} \varepsilon_{d}} ; \quad \quad \frac{1}{G_{(r)}}= \begin{cases}1,355 r^{1,566} & (\mathrm{r} \leq 0,824) \\
1 & (\mathrm{r}>0,824)\end{cases} \\
\left(\frac{V-V_{D} X_{D}}{V_{d}}\right)^{2}+2 G_{(r)}\left(\frac{V-V_{D} X_{D}}{V_{d}}\right)\left(\frac{V-X_{D}-V_{d} X_{d}}{V_{D}-1}\right)+\left(\frac{V-X_{D}-V_{d} X_{d}}{V_{D}-1}\right)^{2}=1\end{array}$ \\
\hline
\end{tabular}

Fonte: Autores.

\subsection{Simulação empregando método de elementos discretos (DEM)}

A metodologia DEM e suas variantes estão bem estabelecidas e são descritas em detalhes por Cleary (2008). O modelo de contato, usado para contabilizar as forças de interação entre duas esferas rígidas que pertencem a duas diferentes partículas, é baseado no modelo de Hertz-Mindlin, que caracteriza as interações por meio de três coeficientes: coeficiente de restituição, coeficiente de atrito estático e coeficiente de atrito de rolamento.

Neste trabalho, empregou-se um coeficiente de restituição de 0,9 e a Razão de Poison de 0,25 e o módulo de Young de $100 \mathrm{GPa}$.

Um Planejamento Composto Central, com 4 fatores foi implementado para calibrar os coeficientes de atrito estático e de rolamento partícula-partícula (PP) e partícula-parede (PW). Além de verificar o efeito desses coeficientes sobre a dinâmica das partículas, e consequentemente a porosidade do leito, foi calculado o desvio dos valores simulados e em relação ao experimental a fim de selecionar o conjunto dos coeficientes que representa o comportamento das forças de contato de das 
esferas. Para tal, utilizou-se um sistema monopartículas com EV de 0,004 m. O efeito dos parâmetros sobre a porosidade da mistura foi avaliado empregando a técnica de superfície de resposta,

O software EDEM foi empregado para a realização das simulações de empacotamento de partículas. Foram empregadas partículas de esferas de vidro de 1 e $4 \mathrm{~mm}$ de diâmetro, e com massa específica de $2480 \mathrm{~kg} / \mathrm{m}^{3}$. A geometria do cone a mesma do tronco de cone do leito de jorro, com diâmetro de base de $0,035 \mathrm{~m}$, altura de $0,15 \mathrm{~m}$ e abertura final de 0,21 $\mathrm{m}$ de diâmetro.

Nas simulações do empacotamento de partículas, um plano de injeção foi criado na altura de $0,08 \mathrm{~m}$, para viabilizar a adição das partículas ao leito até que não houvesse mais volume disponível para a inserção de novas partículas. Ao fim da simulação, o número de partículas que compunham o leito foi contabilizado e calculado o fator de empacotamento e a porosidade do leito.

\section{Resultados e discussão}

\subsection{Comportamento experimental da porosidade em função da composição das misturas}

A Figura 3 apresenta o efeito da razão entre os diâmetros $(\delta=\mathrm{d} / \mathrm{D})$ sobre a distribuição de porosidade de acordo com a composição da mistura. As condições de $\delta=0,15$ e $\delta=0,30$ foram reportadas por Yu et al. (1996), empregando esferas de vidro.

Figura 3. Efeito da razão de tamanho entre as partículas $(\delta=\mathrm{d} / \mathrm{D})$ sobre a relação porosidade em função da composição da mistura: comparação dos dados experimentais com os obtidos por Yu et al. (1996).

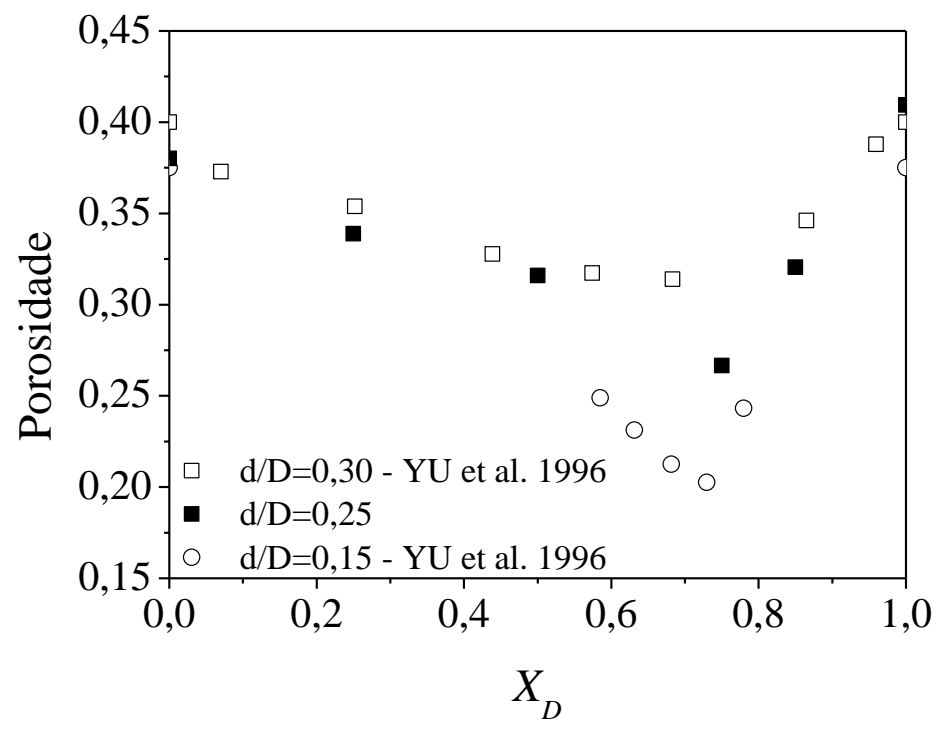

Fonte: Autores.

Observa-se na Figura 3 que a condição estudada neste trabalho, $(\delta=0,25)$ apresenta uma curva de porosidade intermediária entre as demais, mostrando a coerência deste experimento com os dados da literatura.

A porosidade do leito empacotado com EV de $0,004 \mathrm{~m}$ levou a um leito mais poroso $(\varepsilon=0,409)$, enquanto o leito com EV de $0,001 \mathrm{~m}$ se mostrou mais compactado $(\varepsilon=0,38)$, reforçando que o aumento das partículas tende a levar à uma maior fração de espaços vazios entre partículas. No entanto, dentre as composições estudadas, observou-se um valor mínimo de porosidade para misturas com $75 \%$ de EV de $0,004 \mathrm{~m}$ e $25 \%$ de EV de 0,001 m.

A Figura 4 mostra a comparação entre os dados experimentais de porosidade das misturas e os valores preditos pelos Modelos A, B, C e D. 
Com relação ao ajuste de modelos de empacotamento de mistura aos dados experimentais, observa-se na Figura 4 que o modelo $\mathrm{A}$, que não contabiliza nenhum mecanismo de empacotamento, mostrou-se inadequado, e incapaz de prever a composição de mínima porosidade. Os modelos B e D apresentaram resultados similares, mas incapazes de predizer com precisão a porosidade para composições em que a porosidade da mistura é mínima.

Figura 4. Ajuste dos modelos de empacotamento aos dados experimentais de porosidade em função da composição da mistura.

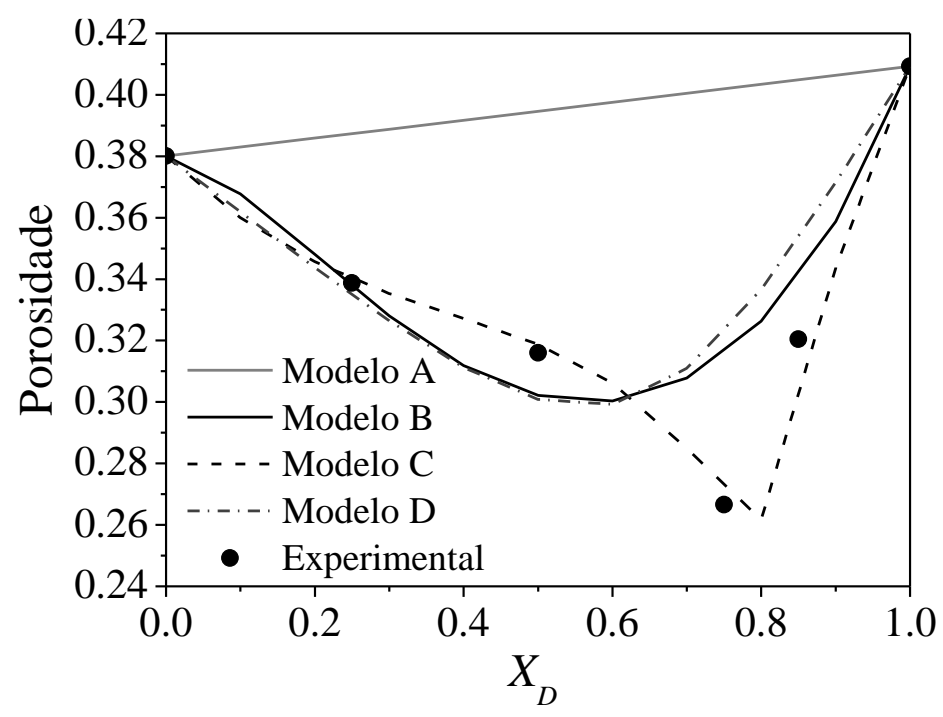

Fonte: Autores.

Observa-se nos resultados da Figura 4, que as funções de correção empregadas no modelo C (Dias et al., 2004) possibilitaram um bom ajuste do modelo aos dados experimentais de porosidade. Provavelmente, isto se deve ao cálculo da composição em que a porosidade é mínima e à correção da equação que antecede essa composição $X_{D} \leq X_{D, \text { min }}$. A região de $X_{D, \min }$ pode ser considerada uma região de transição para o mecanismo de enchimento, em que a estrutura das partículas maiores no leito é preenchida pelas partículas menores.

\subsection{Simulações DEM do empacotamento de misturas}

O Planejamento Composto Central com 27 análises do empacotamento de esferas de vidro de $4 \mathrm{~mm}$ apresentou variação no número de partículas e consequentemente na porosidade (Tabela 2).

De acordo com os dados experimentais, o leito empacotado com esferas de vidro de $4 \mathrm{~mm}$ apresenta porosidade de 0,409, um resultado similar foi obtido de maneira aproximada na simulação de número 18 do planejamento ( $\mu_{s-P P}=0,964$; $\left.\mu_{r-P P}=0,200 ; \mu_{s-P W}=0,200 ; \mu_{r-P W}=0,100\right)$, que apresenta alto valor de coeficiente de atrito estático partícula-partícula $(0,964)$ e as outras variáveis no nível intermediário. 
Tabela 2. Planejamento Composto Central para avaliação dos parâmetros e resultados de porosidade.

\begin{tabular}{|c|c|c|c|c|c|c|}
\hline \multirow[b]{2}{*}{ Simulação } & \multicolumn{4}{|c|}{ Fatores } & \multicolumn{2}{|c|}{ Resposta } \\
\hline & $\begin{array}{c}\mathrm{X}_{1} \\
\left(\mu_{s-P P}\right)\end{array}$ & $\begin{array}{c}\mathrm{X}_{2} \\
\left(\mu_{r-P P}\right)\end{array}$ & $\begin{array}{c}\mathrm{X}_{3} \\
\left(\mu_{s-P W}\right)\end{array}$ & $\begin{array}{c}\mathrm{X}_{4} \\
\left(\mu_{r-P W}\right)\end{array}$ & $\varepsilon_{D E M}$ & $\begin{array}{c}\sigma \\
{[\%]}\end{array}$ \\
\hline 1 & $-1,000(0,200)$ & $-1,000(0,100)$ & $-1,000(0,200)$ & $-1,000(0,040)$ & 0,368 & $-11,280$ \\
\hline 2 & $-1,000(0,200)$ & $-1,000(0,100)$ & $-1,000(0,200)$ & $+1,000(0,160)$ & 0,371 & $-10,099$ \\
\hline 3 & $-1,000(0,200)$ & $-1,000(0,100)$ & $+1,000(0,800)$ & $-1,000(0,040)$ & 0,372 & $-9,945$ \\
\hline 4 & $-1,000(0,200)$ & $-1,000(0,100)$ & $+1,000(0,800)$ & $+1,000(0,160)$ & 0,373 & $-9,507$ \\
\hline 5 & $-1,000(0,200)$ & $+1,000(0,300)$ & $-1,000(0,200)$ & $-1,000(0,040)$ & 0,373 & $-9,703$ \\
\hline 6 & $-1,000(0,200)$ & $+1,000(0,300)$ & $-1,000(0,200)$ & $+1,000(0,160)$ & 0,373 & $-9,594$ \\
\hline 7 & $-1,000(0,200)$ & $+1,000(0,300)$ & $+1,000(0,800)$ & $-1,000(0,040)$ & 0,376 & $-8,856$ \\
\hline 8 & $-1,000(0,200)$ & $+1,000(0,300)$ & $+1,000(0,800)$ & $+1,000(0,160)$ & 0,377 & $-8,556$ \\
\hline 9 & $-1,000(0,200)$ & $-1,000(0,100)$ & $-1,000(0,200)$ & $-1,000(0,040)$ & 0,391 & $-4,631$ \\
\hline 10 & $+1,000(0,800)$ & $-1,000(0,100)$ & $-1,000(0,200)$ & $+1,000(0,160)$ & 0,392 & $-4,393$ \\
\hline 11 & $+1,000(0,800)$ & $-1,000(0,100)$ & $+1,000(0,800)$ & $-1,000(0,040)$ & 0,393 & $-4,057$ \\
\hline 12 & $+1,000(0,800)$ & $-1,000(0,100)$ & $+1,000(0,800)$ & $+1,000(0,160)$ & 0,396 & $-3,411$ \\
\hline 13 & $+1,000(0,800)$ & $+1,000(0,300)$ & $-1,000(0,200)$ & $-1,000(0,040)$ & 0,423 & 3,351 \\
\hline 14 & $+1,000(0,800)$ & $+1,000(0,300)$ & $-1,000(0,200)$ & $+1,000(0,160)$ & 0,424 & 3,588 \\
\hline 15 & $+1,000(0,800)$ & $+1,000(0,300)$ & $+1,000(0,800)$ & $-1,000(0,040)$ & 0,428 & 4,409 \\
\hline 16 & $+1,000(0,800)$ & $+1,000(0,300)$ & $+1,000(0,800)$ & $+1,000(0,160)$ & 0,426 & 3,958 \\
\hline 17 & $-1,547(0,036)$ & $0,000(0,200)$ & $0,000(0,200)$ & $0,000(0,100)$ & 0,345 & $-18,472$ \\
\hline 18 & $+1,547(0,964)$ & $0,000(0,200)$ & $0,000(0,200)$ & $0,000(0,100)$ & 0,410 & 0,265 \\
\hline 19 & $0,000(0,500)$ & $-1,547(0,045)$ & $0,000(0,200)$ & $0,000(0,100)$ & 0,379 & $-7,959$ \\
\hline 20 & $0,000(0,500)$ & $+1,547(0,355)$ & $0,000(0,200)$ & $0,000(0,100)$ & 0,419 & 2,442 \\
\hline 21 & $0,000(0,500)$ & $0,000(0,200)$ & $-1,547(0,036)$ & $0,000(0,100)$ & 0,399 & $-2,448$ \\
\hline 22 & $0,000(0,500)$ & $0,000(0,200)$ & $+1,547(0,964)$ & $0,000(0,100)$ & 0,405 & $-1,054$ \\
\hline 23 & $0,000(0,500)$ & $0,000(0,200)$ & $0,000(0,200)$ & $-1,547(0,007)$ & 0,402 & $-1,765$ \\
\hline 24 & $0,000(0,500)$ & $0,000(0,200)$ & $0,000(0,200)$ & $+1,547(0,193)$ & 0,398 & $-2,716$ \\
\hline 25 & $0,000(0,500)$ & $0,000(0,200)$ & $0,000(0,200)$ & $0,000(0,100)$ & 0,402 & $-1,709$ \\
\hline 26 & $0,000(0,500)$ & $0,000(0,200)$ & $0,000(0,200)$ & $0,000(0,100)$ & 0,403 & $-1,427$ \\
\hline 27 & $0,000(0,500)$ & $0,000(0,200)$ & $0,000(0,200)$ & $0,000(0,100)$ & 0,401 & $-1,954$ \\
\hline
\end{tabular}

Fonte: Autores.

Quanto a análise do efeito das variáveis sobre o empacotamento, observa-se por meio da Equação $10\left(R^{2}=0,9885\right)$ e da superfície de resposta (Figura 5), que o coeficiente de atrito estático partícula-partícula é a variável que mais influencia a operação de empacotamento, seguida pelo coeficiente de atrito de rolamento partícula-partícula e a interação entre eles. Nas simulações realizadas, o coeficiente de atrito de rolamento entre partícula-parede não influenciou o valor da porosidade do leito. 
Figura 5. Porosidade do leito $(\mathrm{dp}=4 \mathrm{~mm})$ em função de $\mathrm{x}_{1}\left(\mu_{s-P P}\right)$ e $\mathrm{x}_{2}\left(\mu_{r-P P}\right)$.

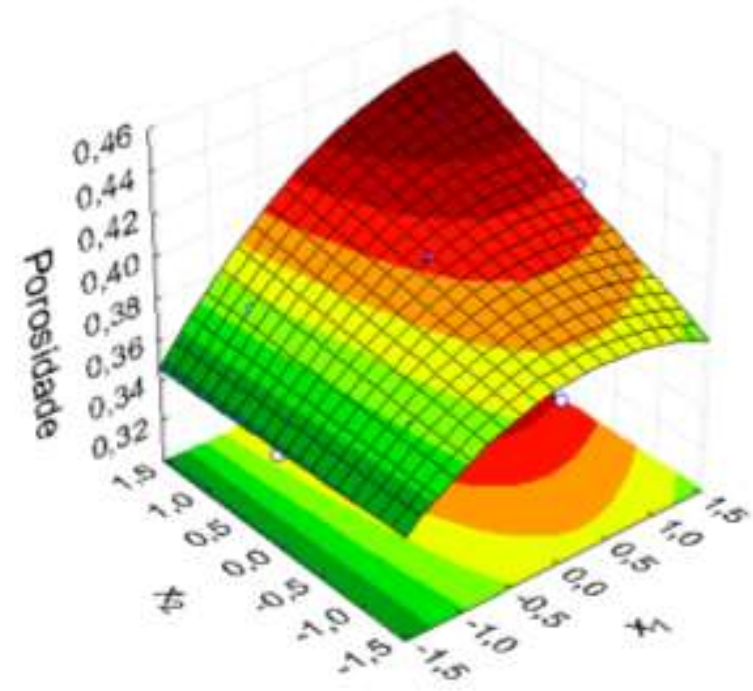

Fonte: Autores.

Após as simulações do leito com monopartículas, realizou-se a simulação correspondente à condição de mínima porosidade, que representa a transição entre os mecanismos de enchimento e ocupação. Para tal, utilizou-se a condição de empacotamento das partículas de $4 \mathrm{~mm}$ e foram adicionadas as partículas de $1 \mathrm{~mm}$ até o preenchimento total dos espaços vazios entre as partículas grandes.

A Figura 6 mostra a comparação da porosidade obtida para as misturas de 1 e $4 \mathrm{~mm}$ obtidas experimentalmente, pelo modelo de Dias et al. (2004) e pela simulação DEM.

Figura 6. Mistura binária de partículas esféricas de 1 e 4 mm: dados experimentais, modelo de Dias et al. (2004) e simulações DEM.

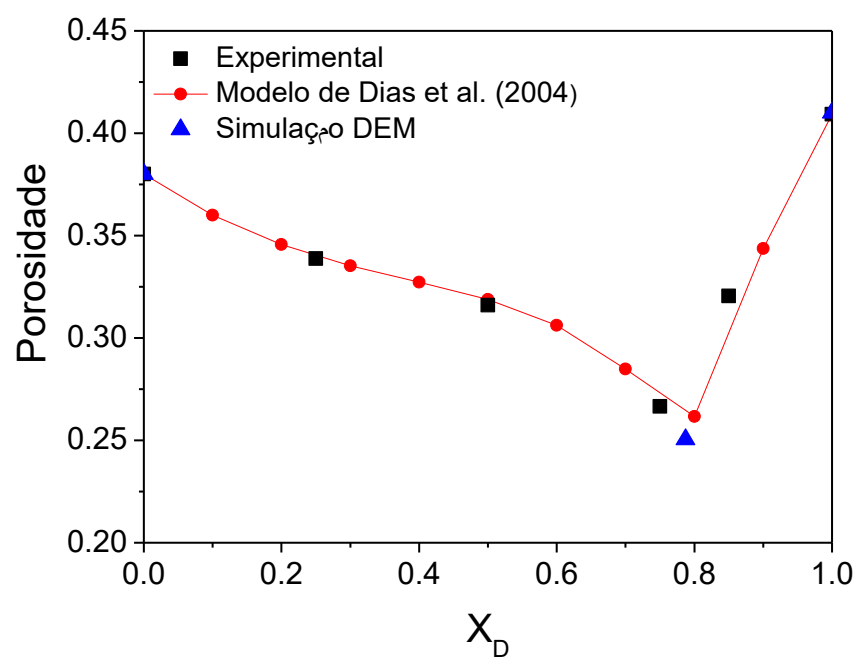

Fonte: Autores.

Infelizmente, os métodos de injeção de partículas testados não foram eficientes quando se trabalhou no regime de ocupação. Além disso, não foi possível realizar as simulações das outras misturas de areia e biomassas, devido ao altíssimo custo computacional das simulações empregando partículas de $1 \mathrm{~mm}$, que chegaram a semanas. 


\section{Conclusão}

Neste trabalho, foram realizados experimentos de empacotamento aleatório de misturas binárias de esferas de vidro de 0,001 e 0,004 m, a fim de estabelecer uma relação de porosidade do leito fixo em função da composição da mistura. Os valores experimentais de porosidade obtidos foram similares ao da literatura. Dentre os modelos testados, o modelo C (Dias et al., 2004) apresentou-se como o mais preciso, provavelmente por aplicar uma função de correção à região que antecede à região de mínima porosidade, que considera um efeito da razão entre as partículas sobre o volume final da mistura.

As simulações DEM se mostraram capazes de representar o empacotamento de sistemas monopartículas e de misturas de partículas, na condição de mínima porosidade. O coeficiente de atrito estático partícula-partícula foi o fator que mais influenciou a porosidade do leito e, portanto, seria adequado calculá-lo de forma experimentalmente precisa.

Como sugestões para trabalhos futuros, tanto os dados de porosidade, como os obtidos pelo modelo C, podem futuramente ser empregados como condição inicial de porosidade para estudos de simulação CFD de leito de jorro operando com mistura de esferas de vidro.

\section{Agradecimentos}

Os autores agradecem o suporte da Capes (AUXPE0856/2015) e Fapemig (PPM-00564-16).

\section{Referências}

Almeida, N. P., Canhadas, M. C., Albertini, M. R. M. C., Santos, K. G. \& Vieira Neto, J. L. (2020). Solid-fluid separation in the gravitational field: Courseware generation using computational fluid dynamics simulation. Computer Applications in Engineering Education, cae.22327, 1-14. https://doi.org/10.1002/cae.22327

Araújo, B. S. A. \& Santos, K. G. (2017). CFD Simulation of Different Flow Regimes of the Spout Fluidized Bed with Draft Plates. Material Science Forum, 899, 89-94. https://doi.org/10.4028/www.scientific.net/MSF.899.89

Batista Júnior, R., Vieira Neto, J. L. \& Santos, K. G. (2019). Estudos de simulação CFD-DEM em um Leito de Jorro Cônico. Revista Brasileira de Ciência, Tecnologia e Inovação, 4, 284-294. https://doi.org/10.18554/rbcti.v4i3.3885

Cleary, P. W. (2008). The effect of particle shape on simple shear flows. Powder Technology, 179, 144-163. https://doi.org/10.1016/j.powtec.2007.06.018

Cundall, P. A. \& Strack, O. D. (1979). A discrete numerical model for granular assemblies. Geotechnique, 29, 47-65. https://doi.org/10.1680/geot.1979.29.1.47

Dias, R. P., Teixeira, J. A., Mota, M. G. \& Yelshin, A. I. (2004). Particulate Binary Mixtures: Dependence of Packing Porosity on Particle Size Ratio. Industrial Engineering Chemistry Research, 43, 7912-7919. https://doi.org/10.1021/ie040048b

Faria, E. V., Sousa, N. G. \& Santos, K. G. (2020). Experimental and numerical study of the heating profile of a solar oven applied to drying. Research, Society and Development, 9(7), e555974368. https://doi.org/10.33448/rsd-v9i7.4368

Graton, L. C. \& Fraser, H. J. J. (1935). Systematic Packing of spheres with particular relation to porosity and permeability. Journal of Geology, 43(8), 785909. https://doi.org/10.1086/624386

Gravena, G. F., Vieira Neto, J. L., Santos, K. G. \& Silvério, B. C. (2019). Estudo da influência dos coeficientes de atrito estático e fricção de rolamento em simulações DEM de tambores rotativos com suspensores. Brazilian Journal of Development, 5, 20800-20811. https://doi.org/10.34117/bjdv5n10-257

Hertz, H. (1882). On the contact of elastic solids. J. reine und angewandte Mathematik, 92, 156-171.

Hlosta, J., Jezerská, L., Rozbroj, J., Žurovec, D., Ne`cas, J. \& Zegzulka, J. (2020). DEM Investigation of the Influence of Particulate Properties and Operating Conditions on the Mixing Process in Rotary Drums: Part 1-Determination of the DEM Parameters and Calibration Process. Processes, 8(2), 222. https://doi.org/10.3390/pr8020222

Lacerda, A. F. M., Vieira, L. G., Nascimento, A. M., Nascimento, S. D., Damasceno, J. J. R. \& Barrozo, M. A. S. (2005). Computational Fluid dynamics techniques for flows in Lapple Cyclone separator. Materials Science Forum, 498-499, 179-185. http://dx.doi.org/10.4028/www.scientific.net/msf.498-499.179

Mangucci, C. B., Stoppe, A. C. R., Morais, A. A., Melo, Y. A., Merola, G. N., Santos, K. G. (2020). Construction of a dust chamber didactic kit and computational fluid dynamics assessment: an active learning practice. Research, Society and Development, 9(11), e41691110069. https://doi.org/10.33448/rsdv9i11.10069

Mindlin, R. D. (1949). Compliance of elastic bodies in contact. J. Appl. Mech., 16(3), 259-268. https://doi.org/10.1115/1.4009973

Mindlin, R. D. \& Deresiewicz, H. (1953). Elastic spheres in contact under varying oblique forces. J. Appl. Mech. Sep 1953, 20(3), 327-344. https://doi.org/10.1115/1.4010702 
Mota, M., Teixeira, J.A., Bowen, W. R., Yelshin, A. (2001). Binary spherical particle mixed beds: porosity and permeability relationship measurement. Transactions of the Filtration Society, 1, 101-106. http://hdl.handle.net/1822/1403

Paula, J. A. A., Faria, E. V., Lima, A. C. P., Vieira Neto, J. L. \& Santos, K. G. (2020). Computational simulation of soybean particles flow in a hopper using computational fluid dynamics (CFD) and discrete elements method (DEM). Research, Society and Development, 9(8), e448985463. https://doi.org/10.33448/rsd-v9i8.5463

Pereira A. S. et al. (2018). Metodologia da pesquisa científica. [free e-book]. Santa Maria/RS, Brasil: UAB/NTE/UFSM.

Rocha, A. A., Stoppe, A. C. R., Silvério, B. C., Santos, K. G. \& Vieira Neto, J. L. (2020). Drying of malt residues in a solar greenhouse and in a fixed bed solar dryer. Research, Society and Development, 9(7), e447974335. https://doi.org/10.33448/rsd-v9i7.4335

Santos, K. G. Aspectos fundamentais da pirólise de biomassa em leito de jorro: Cinética e fluidodinâmica do processo, Tese de doutorado, FEQUI-UFU, Uberlândia-MG, 235p., 2011. https://repositorio.ufu.br/handle/123456789/15058

Santos, K. G., Campos, A. V. P, Ferreira, L. V. \& Barrozo, M. A. S. (2013). Fluid Dynamics of a Sand-Biomass Mixture in a Spouted-Bed Reactor for Fast Pyrolysis. Chemical Engineering \& Technology, 36(12), 2148-2154. https://doi.org/10.1002/ceat.201300356

Santos, K. G., Ferreira, L. V., Santana, R. C. \& Barrozo, M. A. S. (2017). CFD simulation of spouted bed working with a size distribution of sand particles: Segregation aspects. Materials Science Forum, 899, 95-100. https://doi.org/10.4028/www.scientific.net/MSF.899.95

Santos, K. G., Francisquetti, M. C. C., Malagoni, R. A. \& Barrozo, M. A. S. (2015). Fluid Dynamic Behavior in a Spouted Bed with Binary Mixtures Differing in Size. Drying Technology, 33(14), 1746-1757. https://doi.org/10.1080/07373937.2015.1036284

Santos, K. G., Murata, V. V., Barrozo, M. A. S. (2009). Three-dimensional computational fluid dynamics modeling of spouted bed. Canadian Journal of Chemical Engineering, 87(2), 211-219. https://doi.org/10.1002/cjce.20149

Silvério, B. C., Santos, K. G., Duarte, C. R. \& Barrozo, M. A. S. (2014). Effect of the Friction, Elastic, and Restitution Coefficients on the Fluid Dynamics Behavior of a Rotary Dryer Operating with Fertilizer. Ind. Eng. Chem. Res., 53(21), 8920-8926. https://doi.org/10.1021/ie404220h

Stoppe, A. C. R., Vieira Neto, J. L. \& Santos, K. G. (2020). Development of a fixed bed solar dryer: experimental study and CFD simulation. Research, Society and Development, 9(3), e123932667. https://doi.org/10.33448/rsd-v9i3.2667

Tsuji, T., Shibata, T., Yamaguchi, K. \& Uemaki, O. (1989), Mathematical Modelling of Spouted Bed Coal Gasification. Proceeding of the International Conference on Coal Science. Tokyo (Japan), 457-460. https://www.osti.gov/etdeweb/biblio/7271318

Ullmann, G., Gonçalves, S. M., Kyriakidis, Y. N., Barrozo, M. A. S. \& Vieira, L. G. M. (2021). Optimization study of thickener hydrocyclones. Minerals Engineering, 170, 107066. http://doi.org/10.1016/j.mineng.2021.107066

Vieira Neto, J. L., Barrozo, M. A. S., Duarte, C. R., Murata, V. V. (2008), Effect of a draft tube on the fluid dynamics of a spouted bed: Experimental and CFD Studies. Drying Technology, 26(3), 299-307. https://doi.org/10.1080/07373930801897994

Vieira Neto, J. L. ; Costa, D. D. L., Souza, L. V. ; Pires, R. F., Souza, D. L., Silvério, B. C. \& Santos, K. G. (2017). A Fluid Dynamic Study in a Rotating Disk Applied in Granulation of Fertilizers. Materials Science Forum, 899, 142-147. https://doi.org/10.4028/www.scientific.net/MSF.899.142

Yu, A. B., Standish, N. \& Mclean, A. (1993). Porosity Calculation of Binary Mixtures of Nonspherical Particles. Journal of the American Ceramic Society, 76(11), 2813-2816. https://doi.org/10.1111/j.1151-2916.1993.tb04021.x

Yu, A. B., Zou, R. P. \& Standish, N. (1996). Modifying the linear packing model for predicting the porosity of nonspherical particle mixtures. Ind. Eng. Chem. Res., 35(10), 3730-3741. https://doi.org/10.1021/ie950616a

Yu, A. B. \& Standish, N. (1991). Estimation of the porosity of particle mixtures by a linear-mixture packing model, Ind. Eng. Chem. Res., 30(6), 1372-1385. https://doi.org/10.1021/ie00054a045

Yu, A. B., \& Standish, N. (1988). An analytical-parametric theory of the random packing of particles. Powder Technology, 55(3), 171-186. https://doi.org/10.1016/0032-5910(88)80101-3 